\title{
CpG-island promoters drive transcription of human telomeres
}

\author{
SOLOMON G. NERGADZE, ${ }^{1,3}$ BENJAMIN O. FARNUNG ${ }^{2,3}$ HARRY WISCHNEWSKI, ${ }^{2}$ LELA KHORIAULI, ${ }^{1}$ \\ VALERIO VITELLI, ${ }^{1}$ RAGHAV CHAWLA, ${ }^{2}$ ELENA GIULOTTO, ${ }^{1}$ and CLAUS M. AZZALIN ${ }^{2}$ \\ ${ }^{1}$ Dipartimento di Genetica e Microbiologia Adriano Buzzati-Traverso, Università di Pavia, 2700 Pavia, Italy \\ ${ }^{2}$ Institute of Biochemistry, Eidgenössische Technische Hochschule Zürich (ETHZ), CH-8093 Zürich, Switzerland
}

\begin{abstract}
The longstanding dogma that telomeres, the heterochromatic extremities of linear eukaryotic chromosomes, are transcriptionally silent was overturned by the discovery that DNA-dependent RNA polymerase II (RNAPII) transcribes telomeric DNA into telomeric repeat-containing RNA (TERRA). Here, we show that CpG dinucleotide-rich DNA islands, shared among multiple human chromosome ends, promote transcription of TERRA molecules. TERRA promoters sustain cellular expression of reporter genes, are located immediately upstream of TERRA transcription start sites, and are bound by active RNAPII in vivo. Finally, the identified promoter $\mathrm{CPG}$ dinucleotides are methylated in vivo, and cytosine methylation negatively regulates TERRA abundance. The existence of subtelomeric promoters, driving TERRA transcription from independent chromosome ends, supports the idea that TERRA exerts fundamental functions in the context of telomere biology.
\end{abstract}

Keywords: telomeres; TERRA; promoters; transcription; CpG methylation

\section{INTRODUCTION}

Telomeres are heterochromatic DNA-protein complexes located at the end of linear eukaryotic chromosomes. Telomeres are essential to assure genome stability by allowing cells to distinguish between intrachromosomal DNA double-stranded breaks and natural chromosome ends, thereby preventing inappropriate DNA repair events (Palm and de Lange 2008; Xin et al. 2008). Telomeres also set the life span of normal adult somatic cells (Harley et al. 1990; Allsopp et al. 1992; Allsopp and Harley 1995). The DNA component of mammalian telomeres consists of tandem arrays of duplex 5'-TTAGGG-3'/3'-AATCCC-5' repeats, with the G-rich strand extending beyond its complement to form a $3^{\prime}$ overhang; the protein component includes several factors, the most prominent of which are grouped together under the name "shelterin," a multiprotein com-

\footnotetext{
${ }^{3}$ These authors contributed equally to this work.

Reprint requests to: Elena Giulotto, Dipartimento di Genetica e Microbiologia Adriano Buzzati-Traverso, Università di Pavia, 2700 Pavia, Italy; e-mail: elena.giulotto@unipv.it; fax: +39-0382-528496; or Claus M. Azzalin, Institute of Biochemistry, Eidgenössische Technische Hochschule Zürich (ETHZ), CH-8093 Zürich, Switzerland; e-mail: claus.azzalin@bc. biol.ethz.ch; fax: +41-44-6321298.

Article published online ahead of print. Article and publication date are at http://www.rnajournal.org/cgi/doi/10.1261/rna.1748309.
}

plex involved in telomere length regulation and telomere protection (Palm and de Lange 2008; Xin et al. 2008).

In contrast to the longstanding idea that telomeres are transcriptionally silent, we and others recently discovered that RNA polymerase II (RNAPII) transcribes telomeric DNA into telomeric repeat-containing RNA (TERRA) molecules in a variety of eukaryotes including mammals, zebra fish, and budding yeast (Azzalin et al. 2007; Azzalin and Lingner 2008; Chawla and Azzalin 2008; Ho et al. 2008; Luke et al. 2008; Schoeftner and Blasco 2008). As for the majority of RNAPII products, at least a fraction of TERRA is polyadenylated, and RNAPII associates with telomeres in vivo (Azzalin and Lingner 2008; Schoeftner and Blasco 2008). In mammals, TERRA transcripts contain telomeric 5'-UUAGGG-3' RNA repeats, range in size from about 100 bases (b) up to more than 9 kilobases $(\mathrm{kb})$, and are detected exclusively in nuclear cellular fractions (Azzalin et al. 2007; Schoeftner and Blasco 2008). Mammalian TERRA forms discrete nuclear foci that localize to telomeres not only in interphase cells but also in transcriptionally inactive metaphase cells (Azzalin et al. 2007; Ho et al. 2008; Schoeftner and Blasco 2008), suggesting the existence of post-transcriptional mechanisms retaining TERRA at telomeres. Interestingly, some human suppressors with morphogenetic defects in genitalia proteins (namely, SMG1, UPF1, and hEST1A/SMG6), which are effectors of an evolutionarily 
conserved RNA quality control pathway known as nonsense-mediated mRNA decay (Isken and Maquat 2008), negatively regulate TERRA localization to telomeres without affecting TERRA degradation rate or total cellular levels (Azzalin et al. 2007). Short hairpin RNA-mediated depletion of SMG1, UPF1, and hEST1A/SMG6 leads to sudden loss of discrete telomeric tracts (Azzalin et al. 2007), suggesting that dysfunctional TERRA localization may hinder telomere integrity.

In humans, transcription of at least a fraction of TERRA molecules starts within different subtelomeres (the genomic regions immediately adjacent to telomeres) and proceeds toward chromosome ends (Azzalin et al. 2007). Nevertheless, the molecular details of TERRA biogenesis remain to be elucidated. Here we report that $\mathrm{CpG}$ dinucleotide-rich DNA island promoters, sharing conserved repetitive DNA elements, are located on different human chromosome ends and drive transcription of independent TERRA molecules. TERRA CpG-island promoters are able to sustain expression of reporter genes in human cells, are located immediately upstream of TERRA transcription start sites on different subtelomeres, and are bound by active RNAPII in vivo. We also show that DNA methyltransferase (DNMT) 1- and 3b-mediated cytosine methylation of TERRA promoters negatively correlates with RNAPII binding to TERRA promoters and cellular TERRA abundance, suggesting that cytosine methylation represses TERRA promoter transcriptional activity.

\section{RESULTS AND DISCUSSION}

\section{Human subtelomeres contain active CpG-island promoters}

The pro-terminal DNA sequences associated with the longarm telomeres of human chromosomes $\mathrm{X} / \mathrm{Y}(\mathrm{Xq} / \mathrm{Yq})$ and 10 (10q) were isolated nearly 20 years ago and named TelBam3.4 and TelSau2.0, respectively (Brown et al. 1990). The two sequences share a conserved repetitive region that extends for $\sim 1.6 \mathrm{~kb}$ (nucleotides $2110-3117$ ) and $\sim 1.3 \mathrm{~kb}$ (nucleotides 408-1789) until $\sim 280$ nucleotides (nt) upstream of the terminal array in TelBam3.4 and TelSau2.0, respectively (Supplemental Fig. S1). This conserved region contains three different repetitive DNA tracts: the most centromere-proximal tract comprises tandemly repeated 61base-pair (bp) units (five repeats in TelBam3.4 versus six repeats in TelSau2.0); a second, more distal tract comprises 29-bp tandem repeats (nine repeats versus 18 repeats); a third tract comprises five tandemly repeated 37-bp DNA units in both sequences (Supplemental Fig. S1). We refer to the tandem repeat-containing region as "61-29-37 repeats" and to the $\sim 280$ nt comprised between the last 37-bp repeat and the telomeric hexamers as "pre-tel" (Supplemental Fig. S1). BLAST search analysis demonstrated that 61-29-37 repeats are also present at 13 other human subtelomeres
(Supplemental Fig. S2, chromosome arms 1p, 2p, 3q, 4p, 5p, $8 p, 9 p, 11 p, 15 q, 16 p, 17 p, 19 p, 21 q)$, although variable numbers of tandem repeats are observed at different loci. Five additional subtelomeres containing TelBam3.4-like sequences (Supplemental Fig. S2, 3q, 6p, 9q, 12p, 20p) were previously identified using in situ hybridization (Brown et al. 1990). One intrachromosomal locus at 2q13 also contains 61-29-37 repeats (Supplemental Fig. S2) and corresponds to an ancestral telomere-telomere fusion point (Ijdo et al. 1991).

61-29-37 repeats possess a remarkably high $(67 \%-86 \%)$ overall content in $\mathrm{CpG}$ dinucleotides, with a peak corresponding to the 29- and 37-bp repeat tracts (Supplemental Fig. S1). High CpG contents are typical of CpG islands that are found in broad-type promoters of many eukaryotic RNAPII-transcribed genes (Sandelin et al. 2007). Indeed, bioinformatic analysis of TelBam3.4 and TelSau2.0 sequences predicted the existence of $\mathrm{CpG}$-island promoters spanning the 29- and 37-bp repeats (Supplemental Fig. S1). In order to experimentally test the promoter activity of this region, we generated promoter reporter plasmids where progressive $5^{\prime}$ deletions of a $\sim 3.5-\mathrm{kb}$ subtelomeric DNA tract comprising 61-29-37 repeats were inserted upstream of a green fluorescence protein (eGFP) reporter gene (Fig. 1A). Fluorescence microscopy inspection of human HeLa cells transfected with reporter plasmids revealed that a $\sim 1$-kb sequence comprising the 29 - and 37-bp repeat tracts was sufficient to induce cellular eGFP expression (Fig. 1B). Quantification of eGFP fluorescence by densitometric analysis and of eGFP mRNA by quantitative reverse transcription-polymerase chain reaction (qRT-PCR) showed that the identified promoter is approximately five times less efficient than the strong cytomegalovirus (CMV) promoter (Supplemental Fig. S3). Thus, 61-29-37 subtelomeric repeats contain sequences with promoter activity. In agreement with this, active RNAPII is enriched at both 61-29-37 and telomeric repeats over repetitive Alu sequences in vivo, as demonstrated by chromatin immunoprecipitation experiments performed on formaldehyde cross-linked chromatin from U2OS cells using independent antibodies against phosphorylation-activated RNAPII large subunit (Fig. 2; Hirose and Ohkuma 2007).

\section{Subtelomeric CpG-island promoters drive transcription of TERRA molecules}

To determine whether TelBam3.4 and TelSau2.0 subtelomeres are transcribed in vivo, we performed Northern blot analysis of nuclear RNA extracted from human telomerasenegative HLF primary fibroblasts and U2OS tumor cells as well as telomerase-positive HeLa and HEK293T tumor cells, using strand-specific DNA probes, which correspond to TelBam3.4 and TelSau2.0 pre-tel sequences (Fig. 3A). The TelBam3.4 probe is $100 \%$ identical to $\mathrm{Xq} / \mathrm{Yq}$ pre-tels; $92 \%-98 \%$ identical to $8 \mathrm{p}, 9 \mathrm{p}, 15 \mathrm{q}$, and $19 \mathrm{p}$ pre-tels; and 
A

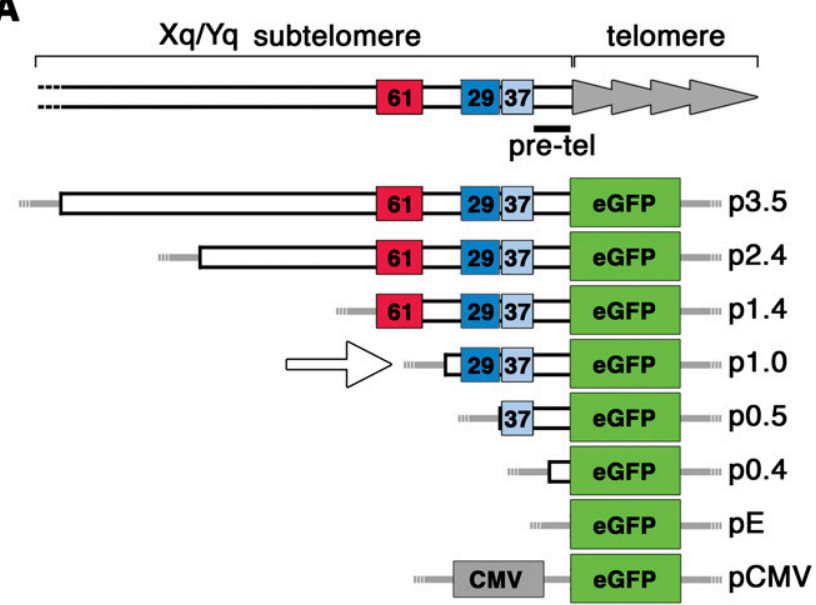

B

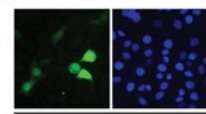

p3.5

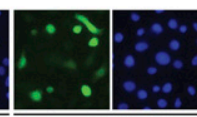

p2.4

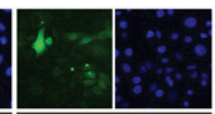

p1.4
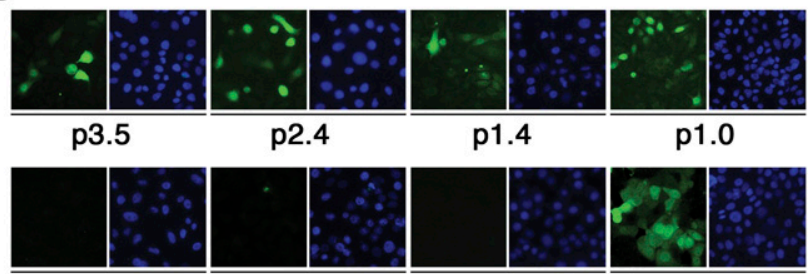

p0.5

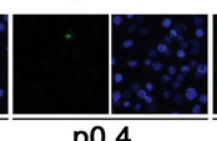

p0.4

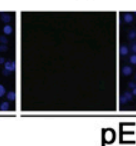

p1.0

FIGURE 1. Promoter reporter assays. (A) A $\sim 3.5$-kb DNA fragment from $\mathrm{Xq} / \mathrm{Yq}$ subtelomeres was cloned upstream of an eGFP cDNA (green box). The fragment contains five 61-bp tandem repeats (red box), ten 29-bp repeats (dark blue box), and five 37-bp repeats (light blue box). The original reporter plasmid was digested with restriction enzymes in order to generate plasmids containing serial $5^{\prime}$ deletions. Plasmid names are given on the right. pE: promoter-less eGFP negative control plasmid; pCMV: cytomegalovirus promoter eGFP positive control plasmid. Gray lines indicate plasmid backbone sequences. The arrow points to the reporter plasmid containing the minimal DNA tract sufficient to induce eGFP expression. (B) Reporter plasmids containing the puromycin resistance gene were transfected into HeLa cells. Puromycin was added $24 \mathrm{~h}$ after transfection, and $4 \mathrm{~d}$ later cells were fixed and imaged by fluorescence microscopy to detect eGFP expression (green, left panels) and DAPIstained DNA (blue, right panels).

90\%-91\% identical to $10 \mathrm{q}$ and $21 \mathrm{q}$ pre-tels. The TelSau2.0 probe is $100 \%$ identical to $10 \mathrm{q}$ pre-tel sequences and $89 \%$ $95 \%$ identical to $8 \mathrm{p}, 9 \mathrm{p}, 16 \mathrm{p}, 15 \mathrm{q}, 19 \mathrm{p}$, and $21 \mathrm{q}$ pre-tels. The two probes are therefore expected to detect partially overlapping families of transcripts originating from a multitude of chromosome ends. After high-stringency washes, both probes detected RNA species varying in length from $\sim 500 \mathrm{~b}$ up to more than $5 \mathrm{~kb}$ in the different cell lines tested (Fig. 3B; Supplemental Fig. S4). The radioactive signals were completely abolished upon ribonuclease A treatment (data not shown), confirming that they originated from RNA. We obtained smeary patterns of hybridization also when we hybridized the same filters with telomeric probes, although the telomeric signal extended to molecular weights lower than 100 b (Fig. 3B; Supplemental Fig. S4). These results suggest that transcripts originating from TelBam3.4 and TelSau2.0 subtelomeres may constitute a fraction of TERRA molecules. It is probable that total cellular TERRA also comprises transcripts deriving from subtelomeres devoid of TelBam3.4 and TelSau2.0 sequences, as well as from 5' processing of TelBam3.4 and TelSau2.0 RNA molecules. Interestingly, while the signal detected with TelBam3.4 and TelSau2.0 probes remains fairly constant throughout all tested cell lines, the signal detected using telomeric probes reveals a two- to eightfold increase in total TERRA levels in U2OS cells, suggesting that in these cells a substantial fraction of TERRA transcripts does not contain TelBam3.4 and TelSau2.0 sequences. Although the molecular mechanisms leading to increased TERRA levels in U2OS remain unclear, a connection might exist between TERRA cellular expression and the homologous recombination-based processes maintaining telomere length in U2OS cells (Royle et al. 2008).

To verify whether TelBam3.4 and TelSau2.0 subtelomeres are indeed transcribed into TERRA molecules, we employed an RT-PCR protocol previously developed to detect TERRA transcripts (Azzalin et al. 2007). We reverse transcribed nuclear RNA using a telomeric $5^{\prime}$-(CCCTAA $)_{5}-3^{\prime}$ oligonucleotide (Fig. 3A, oC; Supplemental Table S1), complementary to the $5^{\prime}$-(UUAGGG) ${ }_{\mathrm{n}}-3^{\prime}$ sequence present in TERRA molecules. We then PCR-amplified the obtained CDNA with unique primer pairs corresponding to TelBam3.4 and TelSau2.0 pre-tel sequences immediately adjacent to the telomeric tracts in the genome (Fig. 3A, o1, o2; Supplemental Table S1). We obtained amplicons matching in size (Fig. 3C) and sequence (data not shown) the ones obtained in PCR reactions performed on genomic DNA, thus proving that the tested subtelomeres are indeed transcribed and that transcription continues into the
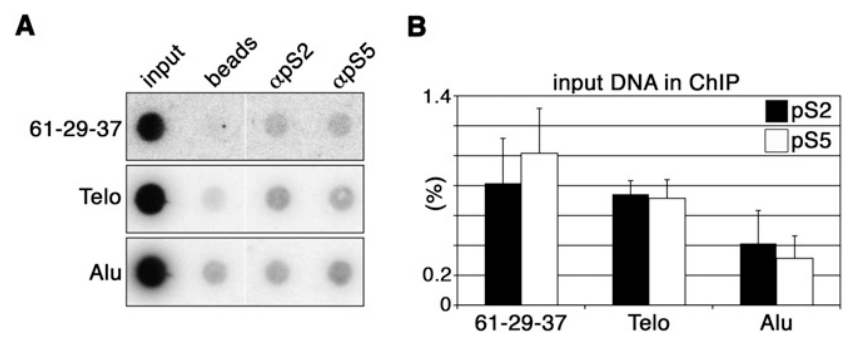

FIGURE 2. RNAPII binds to 61-29-37 repeats in vivo. (A) Formaldehyde cross-linked chromatin from U2OS cells was immunoprecipitated using antibodies raised against phosphorylated serine 2 (pS2) and serine 5 (pS5) from the C-terminal repeat of human RNAPII. Immunoprecipitated DNA was dot-blotted and hybridized with radioactive DNA probes detecting 61-29-37 repeats. The same blot was stripped and re-hybridized sequentially to detect repetitive telomeric (positive control) and Alu sequences. (B) The bar graph shows the fraction of input DNA immunoprecipitated in the different samples, after subtraction of the background signal measured for control reactions performed using only beads. Bars and error bars represent averages and standard deviations from three independent immunoprecipitations. 

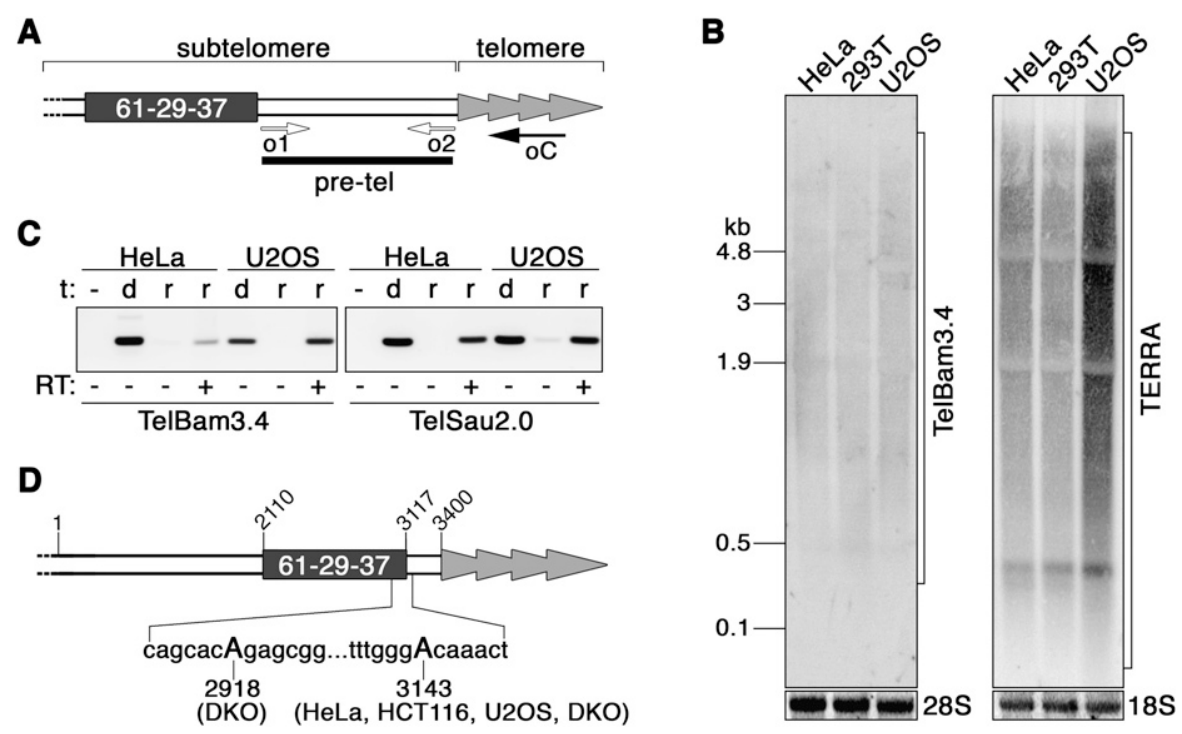

FIGURE 3. TERRA transcription from TelBam3.4 and TelSau2.0 subtelomeres. (A) Schematic representation of a 61-29-37 repeat-containing chromosome end. Note that the sketch is not to scale. $(B)$ Nuclear RNA extracted from the indicated cell lines was electrophoresed and hybridized with radioactive strand-specific probes corresponding to TelBam3.4 pre-tel sequences (black bar in $[A]$ ). The same blot was stripped and probed for total TERRA. Ethidium bromide-stained $28 \mathrm{~S}$ and $18 \mathrm{~S}$ rRNA transcripts are shown to confirm equal RNA loading. Standard molecular weights are on the left in kilobases $(\mathrm{kb}) .(C)$ Nuclear RNA (r) from the indicated cell lines was reverse transcribed (RT) using oC oligonucleotides, and cDNA was PCR amplified using o1 and o2 oligonucleotides specific for TelBam3.4 or TelSau2.0 pre-tel sequences (oligonucleotide positions are indicated by arrows in $A$ ). PCR products were run in agarose gels and stained. Control PCR reactions were performed using genomic DNA (d) as template $(\mathrm{t}) .(D)$ RACE experiments performed on nuclear RNA from HeLa, U2OS, and HCT116 cells and from HCT116-derived cells knockedout for DNA methyltransferases 1 and $3 \mathrm{~b}$ (double $\mathrm{KO}$, DKO) identified the capitalized adenines as transcription start sites at TelBam3.4 subtelomeres. Numbers indicate nucleotide positions as they appear in the database entry M57752.1.

telomeric tract. To reinforce these findings, we annealed nuclear RNA prepared from telomerase positive HCT116 human cancer cells with a TelSau2.0 DNA oligonucleotide adjacent to the telomeric tracts in the genome (Fig. 3A, o2; Supplemental Table S1). The oligonucleotide is $100 \%$ and $70 \%$ complementary to sequences from TelSau2.0 and TelBam3.4 pre-tel transcripts, respectively. We then treated the RNA with RNase $\mathrm{H}$, which digests specifically RNA molecules engaged in DNA/RNA hybrids, and subjected the digested RNA to Northern blot analysis with radioactive telomeric probes. Quantitative analysis of the obtained hybridization profiles reproducibly revealed that the RNase $\mathrm{H}$ treatment led to a shift of the telomeric hybridization profile toward lower molecular weights (Fig. 4). Altogether, these results indicate that TERRA molecules originating from TelBam3.4 and TelSau2.0 subtelomeres exist in different human cell lines and constitute a substantial fraction of total TERRA.

We then used rapid amplification of cDNA ends (RACE) to isolate intact $5^{\prime}$ ends of TelBam3.4 transcripts from cDNA obtained by reverse transcription of HeLa, HLF, HCT116, and U2OS nuclear RNA with the telomeric oC oligonucleotide. Our RACE protocol allows amplification of RNA molecules with a methylated $5^{\prime}$ cap only, thereby preventing detection of uncapped and degraded RNA species. In all cell lines, we identified one unique transcription start site located $27 \mathrm{nt}$ downstream from the last 37-bp repeat (Fig. 3D) and conserved on chromosomes 8p, 9p, $15 \mathrm{q}, 16 \mathrm{p}$, and $19 \mathrm{p}$, and $\mathrm{Xq} / \mathrm{Yq}$ subtelomeres. Although our extensive analysis of RACE products revealed only one transcription start site, we cannot exclude the possibility that alternative- -perhaps more rare- - start sites might exist in the same cells. In addition, as described below, usage of alternative transcription start sites can occur in DNA methyltransferase-deficient cells. Nevertheless, transcription of a fraction of TERRA molecules begins immediately downstream from 61-29-37 repeats and continues toward chromosome ends. Because the physical distance between the identified transcription start site and the telomeric tract is $\sim 250$ nt (Fig. 3D), while the length of TelBam3.4 transcripts visualized by Northern blotting varies between $\sim 500$ and $>5000$ b (Fig. 3B), we infer that transcription can proceed through the telomeric tract for several kilobases.

\section{TERRA promoters are methylated at CpG dinucleotides and cytosine methylation regulates TERRA cellular levels}

DNA methyltransferase (DNMT)-mediated methylation of cytosines at promoter $\mathrm{CpG}$ dinucleotides negatively regulates transcriptional activity (Esteller 2007; Suzuki and Bird 2008). Furthermore, DNMT3b-deficient human cell lines established from patients affected by the immunodeficiency, 
centromere instability, and facial anomalies (ICF) syndrome are characterized by hypomethylated subtelomeric DNA and increased TERRA expression, compared to normal cells from healthy individuals (Yehezkel et al. 2008). Promoter CpG methylation may thus regulate TERRA expression. We digested HLF, HeLa, and U2OS genomic DNA with the methylation-sensitive HpaII restriction enzyme or with its methylation-insensitive isoschizomer MspI and performed Southern blot hybridizations with radiolabeled DNA probes, corresponding to 6129-37 repeats. While MspI digestion generated prominent hybridization bands ranging in size between 100 and $500 \mathrm{bp}$, additional bands of higher molecular weight (up to more than $23 \mathrm{~kb}$ ) appeared in HpaII-digested samples (Fig. 5A). This indicates that 61-29-37 repeats are methylated in vivo. 61-29-37 repeat methylation appears to be nearly complete in HeLa cells, while a lower degree of methylation is observed in HLF and U2OS cells, as demonstrated by increased size heterogeneity of the hybridization bands in HpaII-digested samples (Fig. 5A). This is consistent with recent observations showing that telomerase-positive human cancer cells carry hypermethylated subtelomeric DNA compared to telomerase-negative cancer or primary human cells (Vera et al. 2008; Ng et al. 2009; Tilman et al. 2009). We then analyzed the methylation state of 61-29-37 repeats in human HCT116 cells knocked-out for DNMT1 $\left(D N M T 1^{-/-}\right)$, for DNMT3b $\left(D N M T 3 b^{-/-}\right)$or for both (double $\mathrm{KO}[\mathrm{DKO}]$ ) methyltransferases (Rhee et al. 2000; Rhee et al. 2002). Concomitant disruption of DNMT1 and DNMT3b genes completely abolished methylation at 61-2937 repeat $\mathrm{CpG}$ dinucleotides, while only modest changes were observed in single $\mathrm{KO}$ cells (Fig. 5B), implying that DNMT1 and DNMT3b enzymes cooperatively maintain DNA methylation at 61-29-37 repeats in HCT116 cells. Northern blot analysis disclosed dramatically increased steady-state levels of TelBam3.4, TelSau2.0, and total TERRA transcripts in DKO cells compared to parental, $\mathrm{DNMT1}^{-/-}$, and DNMT3b ${ }^{-1-}$ cells (Figs. 4, 6A; Supplemental Fig. S5). Similarly, treatments of HeLa cells with the DNMT inhibitor 5-azacytidine (5aza) induced an approximately threefold increase in TelBam3.4 and TelSau2.0 RNA species (Supplemental Fig. S6). The extra TelBam3.4 and TelSau2.0 transcripts detected in DKO cells (Figs. 4, 6A) are, at least in part, true TERRA molecules for the following reasons: first, RT-PCR experiments using telomeric oC oligonucleotides for reverse transcription produced TelBam3.4 and TelSau2.0 amplicons matching the corresponding genomic sequences both in parental and DKO cells (Supplemental Fig. S5). Second, RACE experiments performed on nuclear RNA prepared from DKO cells identified the same TelBam3.4 transcription start site as for parental HCT116 cells (see Fig. 3D, nucleotide 3143) in 31 out of the 41 analyzed positive RACE plasmid clones and a second transcription start site within the first 37-bp repeat (see Fig. 3D, nucleotide 2918) in the remaining 10 clones. Third, RNase $\mathrm{H}$ digestion of DKO nuclear RNA, previously annealed with TelSau2.0 DNA oligonucleotides, induced a substantial shift of the TERRA hybridization profile toward lower molecular weights (Fig. 4). In conclusion, $\mathrm{CpG}$ methylation appears to modulate the cellular abundance of TERRA (including TERRA transcribed from TelBam3.4 and TelSau2.0 subtelomeres) as well as the usage of independent transcription start sites. We hypothesize that CpG methylation represses TERRA promoter transcriptional activity. Consistent with this hypothesis, ChIP experiments revealed increased levels of active

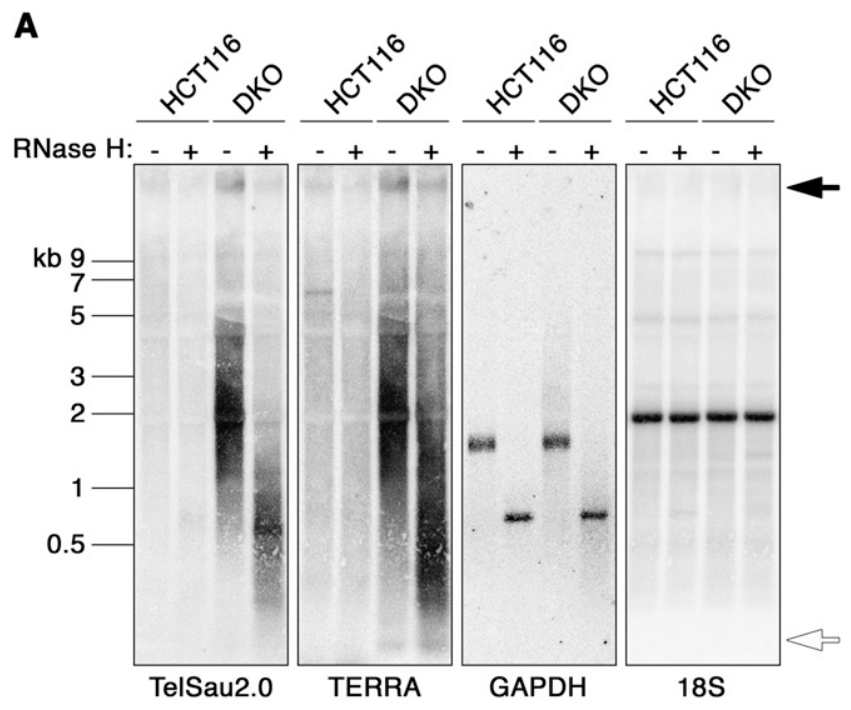

B
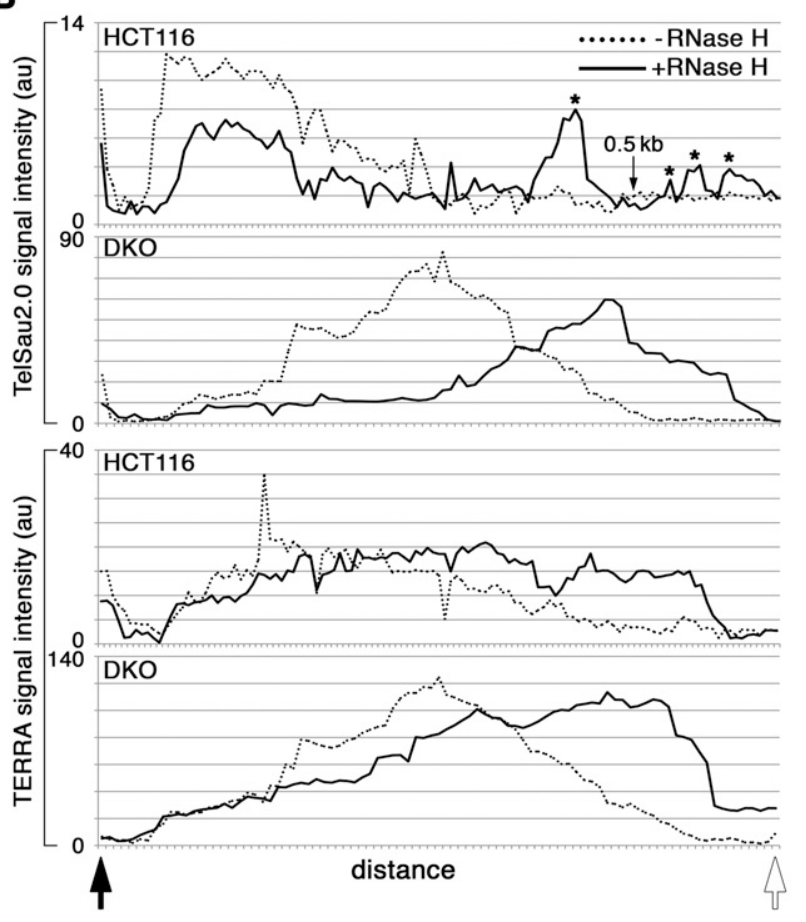

FIGURE 4. (Legend on next page) 
RNAPII binding to 61-29-37 and telomeric repeat DNA in DKO cells compared to parental HCT116 cells (Fig. 6B). However, we cannot exclude the possibility that, in DKO cells, impaired degradation of TERRA transcripts could occur, thereby contributing to their increased steady-state levels.

\section{CONCLUDING REMARKS}

Aberrant cellular accumulation of TERRA transcripts has been reported in Saccharomyces cerevisiae yeast mutants knocked out for the $5^{\prime}-3^{\prime}$ RNA exonuclease Rat $1 p$, which is thought to degrade RNA species derived from transcription occurring past poly(A) cleavage sites in RNAPII-transcribed genes (Rosonina et al. 2006; Luke et al. 2008). One could therefore speculate that TERRA molecules originate from inefficient transcription termination of genes placed subtelomerically. On the contrary, we reveal that in human cells, CpG-island promoters, embedded within subtelomeric 61-29-37 repeats, drive transcription of TERRA molecules possibly from up to 20 different subtelomeres, suggesting that common regulatory mechanisms control the biogenesis of TERRA transcripts originating from independent chromosome ends. We were unable to identify 61-29-37-like repeats at the remaining 26 human subtelomeres, including $11 \mathrm{q}$ and $\mathrm{Xp} / \mathrm{Yp}$ subtelomeres, which were previously demonstrated to be transcribed (Azzalin et al. 2007). It is likely that different promoter types contribute to the biogenesis of total human TERRA, although we cannot exclude the possibility that illdefined subtelomeric sequences available in the databases (Riethman et al. 2004; Riethman 2008) might have led us to underestimate the actual number of human subtelomeres carrying 61-29-37 repeats.

FIGURE 4. Transcripts from TelSau2.0 and TelBam 3.4 subtelomeres constitute a substantial fraction of total TERRA molecules. (A) Nuclear RNA from the indicated cell lines was annealed to a DNA oligonucleotide $100 \%$ and $70 \%$ complementary to TelSau 2.0 and TelBam3.4 pre-tel transcripts, respectively. The same RNA was also annealed to a DNA oligonucleotide complementary to GAPDH mRNA. RNA was incubated with RNase H or left untreated, electrophoresed, and hybridized with radioactive strand-specific probes corresponding to TelSau2.0 pre-tel sequences. The same blot was stripped and probed sequentially to detect total TERRA, GAPDH (positive control for RNAse H reaction), and 18S (control for RNase $\mathrm{H}$ specificity and loading control) RNA sequences. Standard molecular weights are shown on the left in kilobases $(\mathrm{kb})$. (B) Charts showing the distribution of signal intensity for the Northern blots shown in $(A)$. The asterisks indicate RNase $\mathrm{H}$-induced peaks in the TelSau2.0 hybridization profile of HCT116 RNA. The peaks are likely to correspond to TelSau2.0-like pre-tel sequences from which the telomeric RNA tracts have been removed by the RNase $\mathrm{H}$ treatment. The black and white arrows indicate the lane area used for signal quantifications. Similar shifts in the telomeric profiles were obtained when RNA was incubated only with TelSau2.0 oligonucleotides, omitting the GAPDH oligonucleotides from the reactions (data not shown).
A

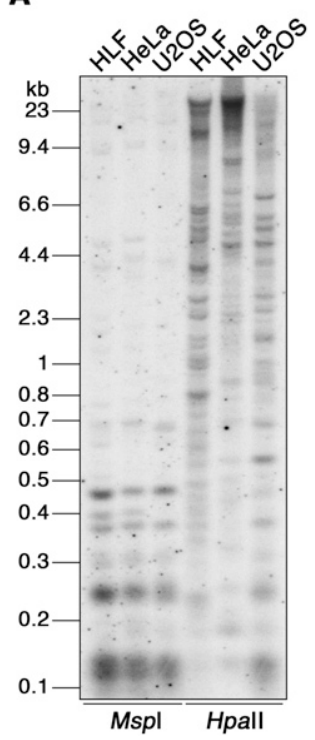

B

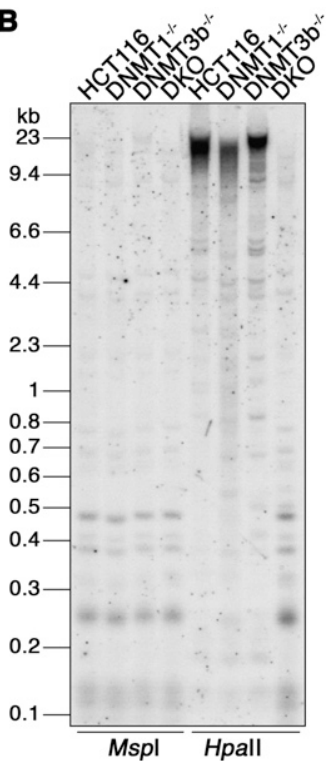

FIGURE 5. Cytosine methylation at 61-29-37 repeats. $(A, B)$ Genomic DNA extracted from the indicated cell lines was digested with the methylation-sensitive HpaII restriction enzyme or with its methylation-insensitive isoschizomer MspI. Digested DNA was electrophoresed, blotted, and hybridized with radioactive DNA probes detecting 61-29-37 repeats. (B) DNMT1 ${ }^{-1-}$ and DNMT36 ${ }^{-1-}$ are HCT116-derived clonal cell lines knocked-out for DNA methyltransferases 1 and $3 \mathrm{~b}$, respectively. Double KO (DKO-) are HCT116-derived clonal cell lines knocked-out for both methyltransferases. Standard molecular weights are shown on the left of each blot in kilobases $(\mathrm{kb})$.

Our discoveries define human telomeres as components of integral "genic" units and make TERRA rise above the transcriptional noise associated with the human genome, supporting the idea that TERRA might exert important functions in telomere biology. It has been proposed that TERRA could repress telomerase activity at chromosome ends by base-pairing with the template sequence in the telomerase RNA moiety (Luke et al. 2008; Schoeftner and Blasco 2008). In this scenario, methylation-mediated transcriptional repression of TERRA CpG-island promoters may be part of an epigenetic tumor suppressor genesilencing program accompanying cell transformation (Esteller 2007; Suzuki and Bird 2008).

\section{MATERIALS AND METHODS}

\section{DNA sequence analysis}

We aligned the TelBam3.4 and TelSau2.0 sequences with the entire human genome using the basic local alignment search tool (BLAST) at the National Center for Biotechnology Information (http://www.ncbi.nlm.nih.gov/blast/Blast.cgi). To analyze $\mathrm{CpG}$ dinucleotide contents and predict $\mathrm{CpG}$ islands, we utilized the CpGPlot/CpGReport at the European molecular biology open software suite program (EMBOSS; http://www.ebi.ac.uk/Tools/emboss/ cpgplot/) (Rice et al. 2000). 
A
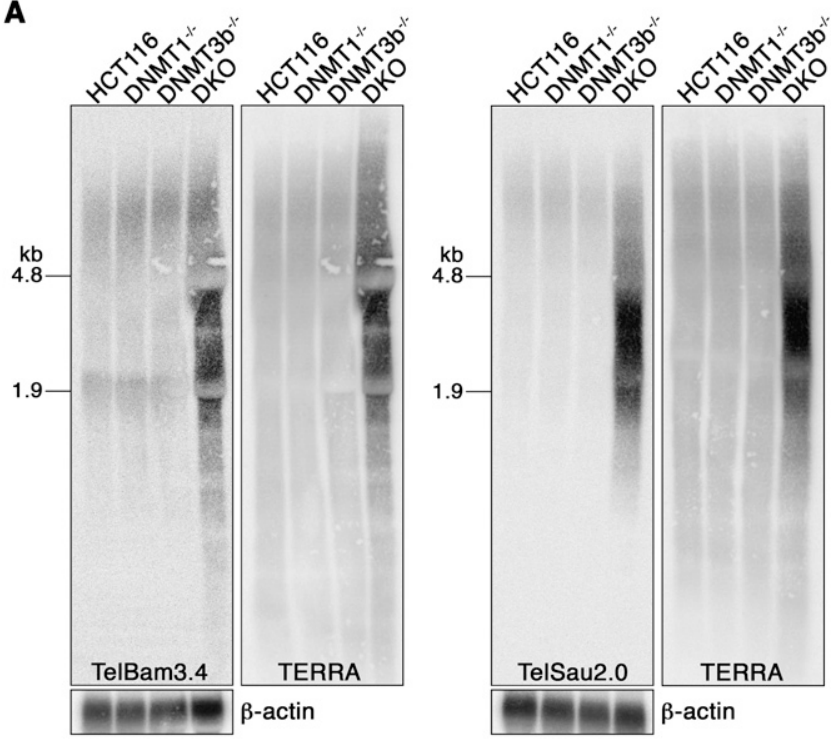

B

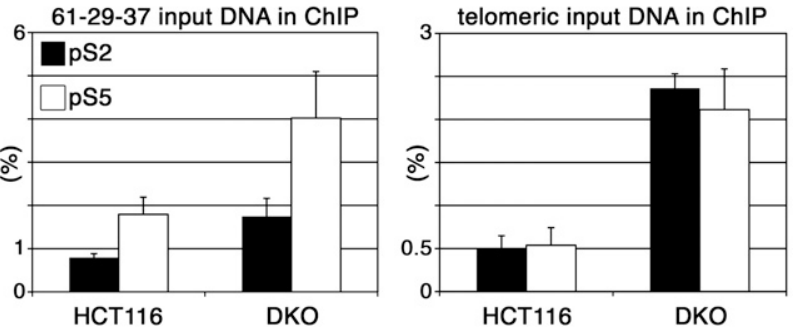

FIGURE 6. DNMT1 and DNMT3b enzymes cooperatively repress TERRA cellular levels. (A) Nuclear RNA was extracted from the indicated cell lines, electrophoresed, blotted, and hybridized using strand-specific TelBam3.4 or TelSau2.0 radioactive probes. The same blots were stripped and re-probed sequentially for total TERRA and $\beta$-actin. Molecular weights of $28 \mathrm{~S}(4.8-\mathrm{kb})$ and $18 \mathrm{~S}(1.9-\mathrm{kb}) \mathrm{rRNA}$ are shown on the left. (B) Formaldehyde cross-linked chromatin from the indicated cell lines was immunoprecipitated using antibodies raised against phosphorylated serine 2 (pS2) and serine 5 (pS5) from the C-terminal repeat of human RNAPII. Immunoprecipitated DNA was dot-blotted and hybridized sequentially with radioactive DNA probes detecting 61-29-37 and telomeric repeats. Graphs show the fraction of input DNA immunoprecipitated in the different samples, after subtraction of the background signal measured for control reactions performed using only beads. Bars and error bars represent averages and standard deviations from three independent immunoprecipitations.

\section{Plasmid constructions}

Since TelBam3.4 and TelSau2.0 plasmids (Brown et al. 1990) were no longer available, we subcloned a $\sim 3-\mathrm{kb} B a m H I-E c o R I$ human genomic DNA fragment from the bacterial artificial chromosome clone RP11-34P13. This fragment is identical to a portion of the TelBam3.4 sequence and contains 61-, 29-, and 37-bp repeats. The pre-tel fragment comprised between the last 37-bp repeat and the telomeric repeats was obtained by PCR on human genomic DNA using primers constructed on the TelBam 3.4 sequence. We constructed a plasmid containing the eGFP cDNA, under the $\mathrm{CMV}$ promoter, and the puromycin resistance gene using the
pEGFN1 and pPUR plasmids (Clontech). We then substituted the CMV promoter with the $\sim 3.5$-kb TelBam3.4 fragment and obtained deletions using appropriate restriction enzymes or substituting the CMV promoter with PCR amplified fragments. We checked all constructs by sequencing.

\section{Cell lines and tissue culture procedures}

We cultured HLF (human lung fibroblasts), HeLa (human cervical carcinoma), HEK293T (human embryonic kidney), U2OS (human osteosarcoma), and HCT116 (human colon carcinoma) cells in high-glucose D-MEM supplemented with $10 \%$ fetal calf serum, nonessential amino acids, and penicillinstreptomycin (Invitrogen). Where indicated, we treated cells with $10 \mu \mathrm{M}$ 5-azacytidine (Sigma Aldrich). For promoter reporter assays, we transfected HeLa cells using the Lipofectamine 2000 reagent (Invitrogen). Twenty-four hours after transfection, we selected positively transfected cells in medium containing $1 \mu \mathrm{g} / \mathrm{mL}$ puromycin for $4 \mathrm{~d}$.

\section{RNA preparation and analysis}

We prepared RNA from whole cells or nuclei-enriched cellular fractions (Azzalin et al. 2007) using the Nucleospin RNA II kit (Macherey-Nagel). We treated RNA twice with RNase-free DNase I (New England Biolabs) to eliminate any DNA contaminations. For RNase H experiments, we mixed $10 \mu \mathrm{g}$ of nuclear RNA with 600 pmol of a TelSau2.0 oligonucleotide and 600 pmol of a GAPDH oligonucleotide (see Supplemental Table S1). We incubated the RNA/DNA mix at $65^{\circ} \mathrm{C}$ for $4 \mathrm{~min}$ and at room temperature for $20 \mathrm{~min}$. We then added $1 \mathrm{U}$ of RNase $\mathrm{H}$ (New England Biolabs) to the mix and allowed digestion at $37^{\circ} \mathrm{C}$ for $1 \mathrm{~h}$. For Northern blots, we electrophoresed 10-20 $\mu$ g of RNA in $1.2 \%$ formaldehyde agarose gels and blotted it to nylon membranes. We hybridized membranes for $\sim 18 \mathrm{~h}$ in Church buffer containing ${ }^{32} \mathrm{P}$-labeled probes at $50^{\circ} \mathrm{C}-64^{\circ} \mathrm{C}$. The strand-specific telomeric probe used to detect total TERRA was described previously (Azzalin et al. 2007). The TelBam 3.4 and TelSau2.0 probes were genomic PCR products obtained with o1 and o 2 oligonucleotides and labeled by primer extension using the 02 oligonucleotide (see Supplemental Table S1). The $\beta$-actin, GAPDH, and $18 \mathrm{~S}$ rRNA probes were $5^{\prime}$ end-labeled DNA oligonucleotides (see Supplemental Table S1). After hybridization, we washed membranes in $0.2-1 \times$ SSC, $0.5 \%$ SDS at the same temperatures used for hybridizations. We detected radioactive signals using a Storm PhosphorImager (GE Healthcare) and quantified them with Quantity One (Bio-Rad) and with ImageQuant (GE Healthcare) software. For quantitative RT-PCR experiments, we reverse transcribed $1 \mu \mathrm{g}$ of total RNA with random hexamers using the SUPERSCRIPT III RNase $\mathrm{H}^{-}$reverse transcriptase (Invitrogen). We PCR amplified the obtained cDNA using eGFP and GAPDH oligonucleotide pairs (see Supplemental Table S1) for $10 \mathrm{sec}$ at $95^{\circ} \mathrm{C}, 20 \mathrm{sec}$ at $60^{\circ} \mathrm{C}$, and $20 \mathrm{sec}$ at $72^{\circ} \mathrm{C}$ ( 45 cycles) using the LightCycler 480 SYBR Green I master mix and instrument (Roche). The RT-PCR-based approach to amplify chromosomespecific TERRA molecules was previously described (Azzalin et al. 2007). We performed 5' RACE experiments with the FirstChoice RLM-RACE Kit (Ambion) using the oC oligonucleotide for reverse transcription and the TelBam3.4 gene-specific oligonucleotides oR1 and oR2 for PCR (see Supplemental Table S1). 
We cloned RACE products into the pDRIVE vector (Qiagen) and sequenced 18 to 41 independent colony plasmids for each cell line.

\section{DNA methylation analysis}

We prepared genomic DNA with the Wizard genomic DNA kit (Promega) and digested it with HpaII (CpG methylation-sensitive) or MspI (CpG methylation-insensitive) restriction enzymes (New England Biolabs). After electrophoresis in 1.2\% agarose gels, we denatured DNA, transferred it to nylon membranes, and hybridized it for $\sim 18 \mathrm{~h}$ at $64^{\circ} \mathrm{C}$ with ${ }^{32} \mathrm{P}$-labeled probes generated by random primer labeling of a $\sim 1$-kb DNA fragment comprising 61-29-37 repeats. We performed post-hybridization stringency washes in $0.2 \times \mathrm{SSC}, 0.5 \% \mathrm{SDS}$ at $64^{\circ} \mathrm{C}$, and detected and quantified radioactive signals as for Northern blots.

\section{Chromatin immunoprecipitation}

We cross-linked cells in $1 \%$ formaldehyde for $30 \mathrm{~min}$ at room temperature. We resuspended cell pellets in $1 \%$ SDS, $10 \mathrm{mM}$ EDTA, and $50 \mathrm{mM}$ Tris- $\mathrm{HCl}(\mathrm{pH} 8)$; sonicated them using a Bioruptor (Diagenode); and diluted extracts in $150 \mathrm{mM} \mathrm{NaCl}$, $20 \mathrm{mM}$ Tris- $\mathrm{HCl}$ ( $\mathrm{pH} \mathrm{8),} \mathrm{1 \%} \mathrm{Triton} \mathrm{X-100,} \mathrm{and} 2$ mM EDTA. We performed immunoprecipitations using rabbit polyclonal antibodies raised against phosphorylated serine S2 or serine S5 (A300654A and A300-655A, Bethyl Laboratories) from the human RNAPII C-terminal repeat. After isolating immunocomplexes using protein $A$ and $G$ beads and purifying immunoprecipitated DNA with the Wizard SV gel and PCR cleanup system (Promega), we dot-blotted DNA onto nylon membranes and hybridized it with ${ }^{32} \mathrm{P}$-labeled 61-29-37 repeat probes as for DNA methylation analysis. After signal detection, we stripped the filters and hybridized them sequentially with ${ }^{32} \mathrm{P}$-labeled probes detecting telomeric and Alu repeat (see Supplemental Table S1) sequences.

\section{SUPPLEMENTAL MATERIAL}

Supplemental material can be found at http://www.rnajournal.org.

\section{ACKNOWLEDGMENTS}

We are grateful to William R.A. Brown for helpful discussions, and to Raffaella Santoro, Bert Vogelstein, Sergio Comincini, and Fiorenzo Peverali for reagents and instruments. The laboratory of E.G. is supported by Fondazione Cariplo (2008-2507), Ministero dell' Università e della Ricerca (PRIN-2006), and Regione Lombardia Progetto Biomedicina. The laboratory of C.M.A. is supported by the Swiss National Science Foundation (3100A0-120090 and PP00P3123356), ETH Zürich (ETH-15 08-1), and Fondazione Cariplo (2008-2507). R.C. is a Swiss National Science Foundation fellow (323500-115044).

Received May 22, 2009; accepted September 3, 2009.

\section{REFERENCES}

Allsopp RC, Harley CB. 1995. Evidence for a critical telomere length in senescent human fibroblasts. Exp Cell Res 219: 130-136.
Allsopp RC, Vaziri H, Patterson C, Goldstein S, Younglai EV, Futcher AB, Greider CW, Harley CB. 1992. Telomere length predicts replicative capacity of human fibroblasts. Proc Natl Acad Sci 89: 10114-10118.

Azzalin CM, Lingner J. 2008. Telomeres: The silence is broken. Cell Cycle 7: 1161-1165.

Azzalin CM, Reichenbach P, Khoriauli L, Giulotto E, Lingner J. 2007. Telomeric repeat containing RNA and RNA surveillance factors at mammalian chromosome ends. Science 318: 798-801.

Brown WR, MacKinnon PJ, Villasante A, Spurr N, Buckle VJ, Dobson MJ. 1990. Structure and polymorphism of human telomere-associated DNA. Cell 63: 119-132.

Chawla R, Azzalin CM. 2008. The telomeric transcriptome and SMG proteins at the crossroads. Cytogenet Genome Res 122: 194201.

Esteller M. 2007. Cancer epigenomics: DNA methylomes and histonemodification maps. Nat Rev Genet 8: 286-298.

Harley CB, Futcher AB, Greider CW. 1990. Telomeres shorten during ageing of human fibroblasts. Nature 345: 458-460.

Hirose Y, Ohkuma Y. 2007. Phosphorylation of the C-terminal domain of RNA polymerase II plays central roles in the integrated events of eucaryotic gene expression. J Biochem 141: 601608.

Ho CY, Murnane JP, Yeung AK, Ng HK, Lo AW. 2008. Telomeres acquire distinct heterochromatin characteristics during siRNAinduced RNA interference in mouse cells. Curr Biol 18: 183187.

Ijdo JW, Baldini A, Ward DC, Reeders ST, Wells RA. 1991. Origin of human chromosome 2: An ancestral telomere-telomere fusion. Proc Natl Acad Sci 88: 9051-9055.

Isken O, Maquat LE. 2008. The multiple lives of NMD factors: Balancing roles in gene and genome regulation. Nat Rev Genet 9: 699-712.

Luke B, Panza A, Redon S, Iglesias N, Li Z, Lingner J. 2008. The Ratlp $5^{\prime}$ to $3^{\prime}$ exonuclease degrades telomeric repeat-containing RNA and promotes telomere elongation in Saccharomyces cerevisiae. Mol Cell 32: 465-477.

Ng LJ, Cropley JE, Pickett HA, Reddel RR, Suter CM. 2009. Telomerase activity is associated with an increase in DNA methylation at the proximal subtelomere and a reduction in telomeric transcription. Nucleic Acids Res 37: 1152-1159.

Palm W, de Lange T. 2008. How shelterin protects mammalian telomeres. Annu Rev Genet 42: 301-334.

Rhee I, Jair KW, Yen RW, Lengauer C, Herman JG, Kinzler KW, Vogelstein B, Baylin SB, Schuebel KE. 2000. CpG methylation is maintained in human cancer cells lacking DNMT1. Nature 404: 1003-1007.

Rhee I, Bachman KE, Park BH, Jair KW, Yen RW, Schuebel KE, Cui H, Feinberg AP, Lengauer C, Kinzler KW, et al. 2002. DNMT1 and DNMT3b cooperate to silence genes in human cancer cells. Nature 416: 552-556.

Rice P, Longden I, Bleasby A. 2000. EMBOSS: The European molecular biology open software suite. Trends Genet 16: 276277.

Riethman H. 2008. Human telomere structure and biology. Annu Rev Genomics Hum Genet 9: 1-19.

Riethman H, Ambrosini A, Castaneda C, Finklestein J, Hu XL, Mudunuri U, Paul S, Wei J. 2004. Mapping and initial analysis of human subtelomeric sequence assemblies. Genome Res 14: 1828.

Rosonina E, Kaneko S, Manley JL. 2006. Terminating the transcript: Breaking up is hard to do. Genes \& Dev 20: 1050-1056.

Royle NJ, Foxon J, Jeyapalan JN, Mendez-Bermudez A, Novo CL, Williams J, Cotton VE. 2008. Telomere length maintenance-an ALTernative mechanism. Cytogenet Genome Res 122: 281-291.

Sandelin A, Carninci P, Lenhard B, Ponjavic J, Hayashizaki Y, Hume DA. 2007. Mammalian RNA polymerase II core promoters: Insights from genome-wide studies. Nat Rev Genet 8: 424436. 
Schoeftner S, Blasco MA. 2008. Developmentally regulated transcription of mammalian telomeres by DNA-dependent RNA polymerase II. Nat Cell Biol 10: 228-236.

Suzuki MM, Bird A. 2008. DNA methylation landscapes: Provocative insights from epigenomics. Nat Rev Genet 9: 465-476.

Tilman G, Loriot A, Van Beneden A, Arnoult N, Londono-Vallejo JA, De Smet C, Decottignies A. 2009. Subtelomeric DNA hypomethylation is not required for telomeric sister chromatid exchanges in ALT cells. Oncogene 28: 1682-1693.
Vera E, Canela A, Fraga MF, Esteller M, Blasco MA. 2008. Epigenetic regulation of telomeres in human cancer. Oncogene 27: 6817-6833.

Xin H, Liu D, Songyang Z. 2008. The telosome/shelterin complex and its functions. Genome Biol 9: 232.

Yehezkel S, Segev Y, Viegas-Pequignot E, Skorecki K, Selig S. 2008. Hypomethylation of subtelomeric regions in ICF syndrome is associated with abnormally short telomeres and enhanced transcription from telomeric regions. Hum Mol Genet 17: 27762789. 

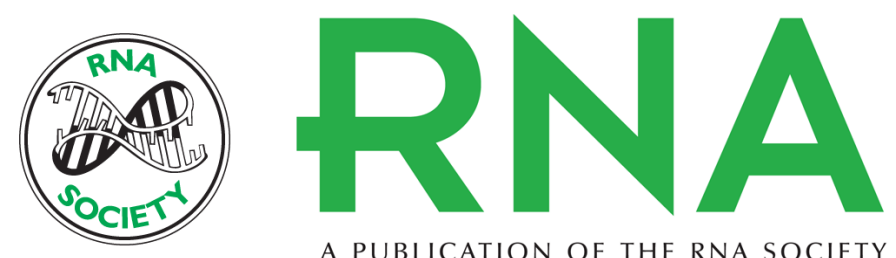

A PUBLICATION OF THE RNA SOCIETY

\section{CpG-island promoters drive transcription of human telomeres}

Solomon G. Nergadze, Benjamin O. Farnung, Harry Wischnewski, et al.

RNA 2009 15: 2186-2194 originally published online October 22, 2009

Access the most recent version at doi:10.1261/rna.1748309

Supplemental

Material

References

\section{License}

Email Alerting

Service
http://rnajournal.cshlp.org/content/suppl/2009/10/02/rna.1748309.DC1

This article cites 29 articles, 5 of which can be accessed free at: http://rnajournal.cshlp.org/content/15/12/2186.full.html\#ref-list-1 